CDAM Research Report LSE-CDAM-2008-07 (18 pp)

\title{
Pricing of contingent claims in a two-dimensional model with random dividends*
}

\author{
Pavel V. Gapeev ${ }^{\dagger} \quad$ Monique Jeanblanc ${ }^{\ddagger}$
}

We study a model where two assets are paying dividends with rates changing from one fixed value to another when any credit event occurs. The credit events are associated with the first times when the asset values fall to some given constant levels. The behavior of asset values is described by exponential diffusion processes with random drift rates and independent driving Brownian motions. We obtain closed form expressions for the ex-dividend prices of certain barrier-type contingent claims with structure similar to firstand second-to-default options in credit risk theory.

\section{Introduction}

One of the known advantages of structural modeling is the explicit form of the credit events that are associated with the passage times of the related asset value processes on some given

*This research benefited from the support of the 'Chaire Risque de Crédit', Fédération Bancaire Française.

${ }^{\dagger}$ London School of Economics, Department of Mathematics, Houghton Street, London WC2A 2AE, United Kingdom; e-mail: p.v.gapeev@lse.ac.uk (supported by ESF AMaMeF Short Visit Grant 1356)

${ }^{\ddagger}$ Université d’Evry Val d’Essonne, Département de Mathématiques, rue Jarlan, F-91025 Evry Cedex, France; Europlace Institute of Finance; e-mail: monique.jeanblanc@univ-evry.fr

Mathematics Subject Classification 2000: Primary 91B70, 60J60, 60G40. Secondary 91B28, 60J25.

Key words and phrases: Structural approach, Brownian motion, running minimum process, first passage time, random dividend rates, dependent credit events, first- and second-to-default options, strong Markov property, full and partial information.

Date: June 18, 2008 
lower barriers. Initiated by Merton [10] and Black and Cox [2], the passage time approach for credit risk theory was further developed and, as a result, a number of subsequent generalizations was obtained (see, e.g., Bielecki and Rutkowski [1; Chapters II and III] or Schönbucher [14; Chapter IX] for an extensive overview). In the present paper, we study a first passage time model for two assets paying dividends, which rates are random and change in time from one fixed value to another. The times of change of dividend rates are assumed to be the first times, when the asset values reach some given lower constant barriers. Such a model corresponds to the situation where the fall of one of the asset values impacts the change of dividend rates not only of the same asset but of the other ones as well. This may happen, for example, with one firm having several branches, where the change of the dividend rate of one of the branches makes an influence on the dividend policy not of the same branch but of the other ones as well. The obtained structure of dependent credit events can also be a contribution to the wide range of first passage time models with dependent defaults (see, e.g., Zhou [17], Giesecke [5], Overbeck and Schmidt [11], Valužis [16] and also [1; Chapter X] or [14; Chapter X] for further references). Note that some other models with random dividends were earlier considered in the literature (see, e.g., Geske [4]).

The purpose of the present paper is to derive the ex-dividend prices of certain barrier-type contingent claims, which structure is similar to the so-called first- and second-to-default options, in the models where the information is generated by both asset values and by the value of one of the assets only. The risk-neutral dynamics of the asset values is modeled by geometric Brownian motions with random drift rates changing their fixed values from one to another at the first times when the value processes fall to some constant levels during the allowed infinite time horizon. For simplicity of exposition, we restrict our consideration to a two-dimensional case and assume that the driving Brownian motions are independent. The prices of the claims are expressed through the transition density of the joint marginal distribution of linearly drifted Brownian motion with its running minimum and the density of its first passage time on a constant level. The consideration of dependent driving Brownian motions would lead to more complicated and less explicit formulas (see, e.g., Iyengar [7], He et al. [6] or Patras [12]). The results of the paper can be naturally extended to the case of several assets, which value processes are driven by independent Brownian motions with random drift rates. 
The paper is organized as follows. In Section 2, we introduce the described above twodimensional structural model. We also recall expressions for the joint transition density of a linearly drifted Brownian motion with its running minimum and for the density of the first time when it hits a constant level. These explicit expressions are used for the derivation of subsequent formulas. In Sections 3 and 4, we evaluate the ex-dividend prices of contingent claims having the structure similar to first- and second-to-default options with respect to the filtration generated by the both asset value processes (full information) and with respect to the filtration generated by one of the processes only (partial information), respectively. The main results of the paper are stated in Theorems 3.1, 3.2, 4.1 and 4.2 .

\section{The model}

In this section we introduce a model with two asset processes paying random dividends and solve the problem of pricing derivatives having a structure similar to first- and second-to-default options under full and partial information.

2.1. For a precise formulation of the problem, let us consider a probability space $(\Omega, \mathcal{G}, P)$ with two independent standard Brownian motions $W^{i}=\left(W_{t}^{i}\right)_{t \geq 0}, i=1,2$. Suppose that there exist two processes $X^{i}=\left(X_{t}^{i}\right)_{t \geq 0}, i=1,2$, given by:

$$
X_{t}^{i}=x_{i} \exp \left(\left(r-\frac{\sigma_{i}^{2}}{2}-\delta_{i, 0}\right) t-\left(\delta_{i, 1}-\delta_{i, 0}\right)\left(t-\tau_{1}\right)^{+}-\left(\delta_{i, 2}-\delta_{i, 0}\right)\left(t-\tau_{2}\right)^{+}+\sigma_{i} W_{t}^{i}\right)
$$

where $\left(t-\tau_{i}\right)^{+}=\max \left\{t-\tau_{i}, 0\right\}, r \geq 0$ and $\sigma_{i}, \delta_{i, \ell}, x_{i}$ are some given strictly positive constants for every $i=1,2$ and $\ell=0,1,2$. Assume that the processes $X^{i}, i=1,2$, describe the risk-neutral dynamics of the values of some assets paying dividends, and $\tau_{i}, i=1,2$, are random times to be specified below, at which some credit events occur resulting the changes of dividend rates. In more details, for every $i=1,2$ fixed, the asset number $i$ pays dividends at the rate $\delta_{i, 0}$ until the time $\tau_{1} \wedge \tau_{2}$, at which the first credit event occurs and the dividend rate is changed into $\delta_{i, \ell}$, where $\ell=1$ if $\tau_{1} \wedge \tau_{2}=\tau_{1}$ and $\ell=2$ if $\tau_{1} \wedge \tau_{2}=\tau_{2}$. Then, the asset $i$ pays dividends with the rate $\delta_{i, \ell}$ until the time $\tau_{1} \vee \tau_{2}$, at which the second credit event occurs and the dividend rate is changed into $\delta_{i, 3}=\delta_{i, 1}+\delta_{i, 2}-\delta_{i, 0}$. After the both credit events occur, the asset $i$ pays dividends with the rate $\delta_{i, 3}$. Here $r$ is the interest rate of a riskless banking 
account and $\sigma_{i}$ is the volatility coefficient.

Following the structural approach, let us define the random time $\tau_{i}$ by:

$$
\tau_{i}=\inf \left\{t \geq 0 \mid X_{t}^{i} \leq b_{i}\right\}
$$

where $b_{i}>0$ is a given constant. By construction, $\tau_{i}$ is a stopping time with respect to the natural filtration $\mathcal{G}_{t}=\sigma\left(X_{s}^{1}, X_{s}^{2} \mid 0 \leq s \leq t\right)$ generated by $X^{i}, i=1,2$. Then, the existence of such a pair of processes $\left(X^{1}, X^{2}\right)$ can be easily deduced from the classical diffusion model with dividends by means of standard change-of-measure arguments.

2.2. The purpose of the present paper is to determine the rational (ex-dividend) prices of contingent claims having the following payoff structure. In the first claim, the amount $C\left(X_{T}^{1}, X_{T}^{2}\right)$ is paid at the maturity $T$ if and only if no credit event occurs before the maturity, and the amount $D_{1, i}\left(\tau_{i}, X_{\tau_{i}}^{1}, X_{\tau_{i}}^{2}\right)$ is paid at the time $\tau_{i}$ if the time of first credit event is $\tau_{i}$ and it occurs before the maturity, for every $i=1,2$. In the second claim, the amount $C_{i}\left(X_{T}^{1}, X_{T}^{2}\right)$ is paid at the maturity $T$ if and only if the two credit events occur before the maturity and the time of the second credit event is $\tau_{i}$, and the amount $D_{2, i}\left(\tau_{i}, X_{\tau_{i}}^{1}, X_{\tau_{i}}^{2}\right)$ is paid at time $\tau_{i}$ if the time of the second credit event is $\tau_{i}$ and it occurs before the maturity, for every $i=1,2$. Without loss of generality, we further assume that the payoffs are already discounted by the banking account, that is equivalent to put $r$ equal to zero.

Note that the contingent claims described above have the structure similar to first- and second-to-default options in the credit risk theory. The ex-dividend price processes $V_{t}^{i}, i=1,2$, of such claims are given by:

$$
V_{t}^{1}=E\left[C\left(X_{T}^{1}, X_{T}^{2}\right) I\left(T<\tau_{1} \wedge \tau_{2}\right)+\sum_{i=1}^{2} D_{1, i}\left(\tau_{i}, X_{\tau_{i}}^{i}, X_{\tau_{i}}^{3-i}\right) I\left(\tau_{i} \leq T, \tau_{i}<\tau_{3-i}\right) \mid \mathcal{G}_{t}\right]
$$

and

$$
V_{t}^{2}=E\left[\sum_{i=1}^{2}\left(D_{2, i}\left(\tau_{i}, X_{\tau_{i}}^{i}, X_{\tau_{i}}^{3-i}\right)+C_{i}\left(X_{T}^{i}, X_{T}^{3-i}\right)\right) I\left(\tau_{3-i}<\tau_{i}<T\right) \mid \mathcal{G}_{t}\right]
$$

for any $0 \leq t \leq T$, respectively (see, e.g., [1; Chapter $\mathrm{X}]$ or $[14$; Chapter $\mathrm{X}]$ ). Here the expectations are taken with respect to the martingale measure and $I(\cdot)$ denotes the indicator function. We further assume that $C\left(x_{1}, x_{2}\right), C_{i}\left(x_{i}, x_{3-i}\right)$ and $D_{k, i}\left(t, x_{i}, x_{3-i}\right), k, i=1,2$, are nonnegative functions such that the integrals appearing below are well defined. For example, 
for the European basket call or put option with the strike price $K>0$ we may set $C\left(x_{1}, x_{2}\right)$ and $C_{i}\left(x_{i}, x_{3-i}\right)$ being equal to $\left(\alpha_{1} x_{1}+\alpha_{2} x_{2}-K\right)^{+}$or $\left(K-\alpha_{1} x_{1}-\alpha_{2} x_{2}\right)^{+}$with some $\alpha_{i} \geq 0, i=1,2$, respectively. We may also assume linear recovery by setting $D_{k, i}\left(t, x_{i}, x_{3-i}\right)=\gamma+\beta_{1} x_{1}+\beta_{2} x_{2}$ with some $\gamma \geq 0$ and $\beta_{i} \geq 0$ for $k, i=1,2$.

Moreover, we shall also determine the prices of the claims under the assumption that the information available on the market is generated by one of the assets only. In that case, the rational prices $V_{t}^{i, j}, i=1,2$, of the claims are given by:

$$
V_{t}^{1, j}=E\left[C\left(X_{T}^{1}, X_{T}^{2}\right) I\left(T<\tau_{1} \wedge \tau_{2}\right)+\sum_{i=1}^{2} D_{1, i}\left(\tau_{i}, X_{\tau_{i}}^{i}, X_{\tau_{i}}^{3-i}\right) I\left(\tau_{i} \leq T, \tau_{i}<\tau_{3-i}\right) \mid \mathcal{G}_{t}^{j}\right]
$$

and

$$
V_{t}^{2, j}=E\left[\sum_{i=1}^{2}\left(D_{2, i}\left(\tau_{i}, X_{\tau_{i}}^{i}, X_{\tau_{i}}^{3-i}\right)+C_{i}\left(X_{T}^{i}, X_{T}^{3-i}\right)\right) I\left(\tau_{3-i}<\tau_{i}<T\right) \mid \mathcal{G}_{t}^{j}\right]
$$

for any $0 \leq t \leq T$, respectively. Here $\mathcal{G}_{t}^{j}=\sigma\left(X_{s}^{j} \mid 0 \leq s \leq t\right)$ is the natural filtration of the process $X^{j}$ for every $j=1,2$.

2.3. For the process $X^{i}$ let us introduce the corresponding running minimum process $M^{i}=\left(M_{t}^{i}\right)_{t \geq 0}$ given by:

$$
M_{t}^{i}=\min _{0 \leq s \leq t} X_{s}^{i} \wedge m_{i}
$$

for any $x_{i} \geq m_{i}>b_{i}>0$ fixed. Then, from the structure of (2.2) it is seen that the default time $\tau_{i}$ takes the form:

$$
\tau_{i}=\inf \left\{t \geq 0 \mid M_{t}^{i} \leq b_{i}\right\}
$$

and in order to obtain the initial values of (2.3)-(2.4) and (2.5)-(2.6), we shall put $x_{i}=m_{i}$ for every $i=1,2$.

It thus follows from (2.8) that the event $\left\{\tau_{i}>t\right\}$ can be expressed as $\left\{M_{t}^{i}>b_{i}\right\}$ for any $t \geq 0$, so that the process $X^{i}$ admits the representation:

$$
d X_{t}^{i}=-X_{t}^{i}\left(\delta_{i, 0}+\left(\delta_{i, 1}-\delta_{i, 0}\right) I\left(M_{t}^{1} \leq b_{1}\right)+\left(\delta_{i, 2}-\delta_{i, 0}\right) I\left(M_{t}^{2} \leq b_{2}\right)\right) d t+X_{t}^{i} \sigma_{i} d W_{t}^{i}
$$

with $X_{0}^{i}=x_{i}$. Therefore, we may conclude that $\left(X^{1}, M^{1}, X^{2}, M^{2}\right)=\left(X_{t}^{1}, M_{t}^{1}, X_{t}^{2}, M_{t}^{2}\right)_{t \geq 0}$ is a (time-homogeneous) strong Markov process with respect to the filtration $\left(\mathcal{G}_{t}\right)_{t \geq 0}$. In the sequel, we also use the notation $\tau_{i}=\tau_{i}\left(x_{1}, m_{1}, x_{2}, m_{2}\right)$ for $x_{i} \geq m_{i}>b_{i}>0$ and every $i=1,2$. 
Note that, by means of standard arguments of filtering theory (see, e.g., [9; Chapter IX]), it is shown that the process $X^{i}$ admits the following representation:

$$
d X_{t}^{i}=-X_{t}^{i}\left(\delta_{i, 0}+\left(\delta_{i, i}-\delta_{i, 0}\right) I\left(M_{t}^{i} \leq b_{i}\right)+\left(\delta_{i, 3-i}-\delta_{i, 0}\right) P\left[M_{t}^{3-i} \leq b_{3-i} \mid \mathcal{G}_{t}^{i}\right]\right) d t+X_{t}^{i} \sigma_{i} d \bar{W}_{t}^{i}
$$

with $X_{0}^{i}=x_{i}$, where the innovation process $\bar{W}^{i}=\left(\bar{W}_{t}^{i}\right)_{t \geq 0}$ defined by:

$$
\bar{W}_{t}^{i}=W_{t}^{i}-\frac{\delta_{i, 3-i}-\delta_{i, 0}}{\sigma_{i}} \int_{0}^{t}\left(I\left(M_{s}^{3-i} \leq b_{3-i}\right)-P\left[M_{s}^{3-i} \leq b_{3-i} \mid \mathcal{G}_{s}^{i}\right]\right) d s
$$

is a standard Brownian motion with respect to the filtration $\left(\mathcal{G}_{t}^{i}\right)_{t \geq 0}$ according to P. Lévy's characterization theorem (see, e.g., [13; Chapter IV, Theorem 3.6]).

2.4. Let us introduce the process $X^{i, j}=\left(X_{t}^{i, j}\right)_{t \geq 0}$ defined by:

$$
X_{t}^{i, j}=x_{i} \exp \left(-\left(\delta_{i, j}+\frac{\sigma_{i}^{2}}{2}\right) t+\sigma_{i} W_{t}^{i}\right)
$$

and its running minimum process $M^{i, j}=\left(M_{t}^{i, j}\right)_{t \geq 0}$ given by:

$$
M_{t}^{i, j}=\min _{0 \leq s \leq t} X_{s}^{i, j} \wedge m_{i}
$$

for any $x_{i} \geq m_{i}>b_{i}>0$ and every $i=1,2$ and $j=0,1,2,3$. Observe that from (2.1) and (2.12) it is seen that $X_{t}^{i}=X_{t}^{i, 0}$ holds for all $0 \leq t \leq \tau_{1} \wedge \tau_{2}$, since we have put $r=0$. It is known (see, e.g., [13], [8] or [3]) that the transition density $g_{i, j}$ of the Markov process $\left(X^{i, j}, M^{i, j}\right)$ defined by:

$$
P_{x_{i}, m_{i}}\left[X_{t}^{i, j} \in d z, M_{t}^{i, j} \in d y\right]=g_{i, j}\left(x_{i}, m_{i} ; t, z, y\right) d z d y
$$

admits the representation:

$$
g_{i, j}\left(x_{i}, m_{i} ; t, z, y\right)=\frac{2}{\sigma_{i}^{3} \sqrt{2 \pi t^{3}}} \frac{\ln \left(y^{2} /\left(x_{i} z\right)\right)}{z y} \exp \left(-\frac{\ln ^{2}\left(y^{2} /\left(x_{i} z\right)\right)}{2 \sigma_{i}^{2} t}+\frac{\rho_{i, j}}{\sigma_{i}} \ln \left(z / x_{i}\right)-\frac{\rho_{i, j}^{2} t}{2}\right)
$$

for all $t>0$ and $z \geq y$ with $m_{i} \geq y>0$, and equals zero otherwise. Here $P_{x_{i}, m_{i}}$ denotes probability under the assumption that $\left(X^{i, j}, M^{i, j}\right)$ starts at $\left(x_{i}, m_{i}\right)$, and we set $\rho_{i, j}=-\delta_{i, j} / \sigma_{i}-$ $\sigma_{i} / 2$.

Let us also define the corresponding hitting time $\tau_{i, j}$ having the form:

$$
\tau_{i, j}=\inf \left\{t \geq 0 \mid X_{t}^{i, j} \leq b_{i}\right\}
$$


for every $i=1,2$ and $j=0,1,2$. It is known that the density $h_{i, j}$ of $\tau_{i, j}$ defined by:

$$
P_{x_{i}, m_{i}}\left[\tau_{i, j} \in d t\right]=h_{i, j}\left(x_{i} ; t\right) d t
$$

admits the representation:

$$
h_{i, j}\left(x_{i} ; t\right)=\frac{\ln \left(x_{i} / b_{i}\right)}{\sigma_{i} \sqrt{2 \pi t^{3}}} \exp \left(-\frac{\left(\ln \left(x_{i} / b_{i}\right)+\rho_{i, j} \sigma_{i} t\right)^{2}}{2 \sigma_{i}^{2} t}\right)
$$

for all $t>0$ and $x_{i} \geq m_{i}>b_{i}>0$.

\section{Case of full information}

In this section we compute conditional expectations (2.3) and (2.4).

3.1. Let us begin by computing the terms for the first-to-default (2.3). For this, applying the Markov property of the process $\left(X^{1}, M^{1}, X^{2}, M^{2}\right)$, we get:

$$
\begin{aligned}
& E_{x_{1}, m_{1}, x_{2}, m_{2}}\left[C\left(X_{T}^{1}, X_{T}^{2}\right) I\left(T<\tau_{1} \wedge \tau_{2}\right) \mid \mathcal{G}_{t}\right] \\
& =I\left(t<\tau_{1} \wedge \tau_{2}\right) E_{x_{1}, m_{1}, x_{2}, m_{2}}\left[C\left(X_{T}^{1}, X_{T}^{2}\right) I\left(T<\tau_{1} \wedge \tau_{2}\right) \mid \mathcal{G}_{t}\right] \\
& =I\left(t<\tau_{1} \wedge \tau_{2}\right) E_{X_{t}^{1}, M_{t}^{1}, X_{t}^{2}, M_{t}^{2}}\left[C\left(X_{T^{\prime}}^{1}, X_{T^{\prime}}^{2}\right) I\left(T^{\prime}<\tau_{1}^{\prime} \wedge \tau_{2}^{\prime}\right)\right]
\end{aligned}
$$

where we set $T^{\prime}=T-t$ for each $0 \leq t \leq T$. Here $E_{x_{1}, m_{1}, x_{2}, m_{2}}$ denotes expectation under the assumption that the process $\left(X^{1}, M^{1}, X^{2}, M^{2}\right)$ starts at $\left(x_{1}, m_{1}, x_{2}, m_{2}\right)$ with some $x_{i} \geq m_{i}>$ $b_{i}>0, i=1,2$. In case $\tau_{i}>t$, we put $\tau_{i}^{\prime}=\tau_{i}\left(X_{t}^{1}, M_{t}^{1}, X_{t}^{2}, M_{t}^{2}\right)$. Then, using the fact that the event $\left\{\tau_{i}>t\right\}$ can be represented in the form $\left\{M_{t}^{i}>b_{i}\right\}$ for any $t \geq 0$, we have:

$$
\begin{aligned}
& E_{x_{1}, m_{1}, x_{2}, m_{2}}\left[C\left(X_{T^{\prime}}^{1}, X_{T^{\prime}}^{2}\right) I\left(T^{\prime}<\tau_{1}^{\prime} \wedge \tau_{2}^{\prime}\right)\right] \\
& =E_{x_{1}, m_{1}, x_{2}, m_{2}}\left[C\left(X_{T^{\prime}}^{1}, X_{T^{\prime}}^{2}\right) I\left(M_{T^{\prime}}^{1}>b_{1}, M_{T^{\prime}}^{2}>b_{2}\right)\right] \\
& =E_{x_{1}, m_{1}, x_{2}, m_{2}}\left[C\left(X_{T^{\prime}}^{1,0}, X_{T^{\prime}}^{2,0}\right) I\left(M_{T^{\prime}}^{1,0}>b_{1}, M_{T^{\prime}}^{2,0}>b_{2}\right)\right]
\end{aligned}
$$

where $\tau_{i}^{\prime}=\tau_{i}\left(x_{1}, m_{1}, x_{2}, m_{2}\right)$ and the processes $\left(X^{i, 0}, M^{i, 0}\right), i=1,2$, are defined in (2.12)(2.13) above. Hence, from (3.1) and (3.2) we obtain:

$$
\begin{aligned}
& E_{x_{1}, m_{1}, x_{2}, m_{2}}\left[C\left(X_{T}^{1}, X_{T}^{2}\right) I\left(T<\tau_{1} \wedge \tau_{2}\right) \mid \mathcal{G}_{t}\right] \\
& =I\left(t<\tau_{1} \wedge \tau_{2}\right) \int_{b_{1}}^{\infty} \int_{b_{1}}^{\infty} \int_{b_{2}}^{\infty} \int_{b_{2}}^{\infty} C\left(x_{1}^{\prime}, x_{2}^{\prime}\right) \prod_{\ell=1}^{2} g_{\ell, 0}\left(X_{t}^{\ell}, M_{t}^{\ell} ; T-t, x_{\ell}^{\prime}, m_{\ell}^{\prime}\right) d x_{\ell}^{\prime} d m_{\ell}^{\prime}
\end{aligned}
$$


where the functions $g_{i, 0}, i=1,2$, are given in (2.15) above.

In a similar way, using the Markov property of the process $\left(X^{1}, M^{1}, X^{2}, M^{2}\right)$, we get:

$$
\begin{aligned}
& E_{x_{1}, m_{1}, x_{2}, m_{2}}\left[D_{1, i}\left(\tau_{i}, X_{\tau_{i}}^{i}, X_{\tau_{i}}^{3-i}\right) I\left(\tau_{i} \leq T, \tau_{i}<\tau_{3-i}\right) \mid \mathcal{G}_{t}\right] \\
& =D_{1, i}\left(\tau_{i}, b_{i}, X_{\tau_{i}}^{3-i}\right) I\left(\tau_{i} \leq t, \tau_{i}<\tau_{3-i}\right) \\
& \quad+E_{x_{1}, m_{1}, x_{2}, m_{2}}\left[D_{1, i}\left(\tau_{i}, b_{i}, X_{\tau_{i}}^{3-i}\right) I\left(t<\tau_{i} \leq T, \tau_{i}<\tau_{3-i}\right) \mid \mathcal{G}_{t}\right] \\
& =D_{1, i}\left(\tau_{i}, b_{i}, X_{\tau_{i}}^{3-i}\right) I\left(\tau_{i} \leq t, \tau_{i}<\tau_{3-i}\right) \\
& \quad+I\left(t<\tau_{1} \wedge \tau_{2}\right) E_{X_{t}^{1}, M_{t}^{1}, X_{t}^{2}, M_{t}^{2}}\left[D_{1, i}\left(t+\tau_{i}^{\prime}, b_{i}, X_{\tau_{i}^{\prime}}^{3-i}\right) I\left(\tau_{i}^{\prime} \leq T, \tau_{i}^{\prime}<\tau_{3-i}^{\prime}\right)\right]
\end{aligned}
$$

for $0 \leq t \leq T$ and

$$
\begin{aligned}
& E_{x_{1}, m_{1}, x_{2}, m_{2}}\left[D_{1, i}\left(t+\tau_{i}^{\prime}, b_{i}, X_{\tau_{i}^{\prime}}^{3-i}\right) I\left(\tau_{i}^{\prime} \leq T^{\prime}, \tau_{i}^{\prime}<\tau_{3-i}^{\prime}\right)\right] \\
& =E_{x_{1}, m_{1}, x_{2}, m_{2}}\left[D_{1, i}\left(t+\tau_{i}^{\prime}, b_{i}, X_{\tau_{i}^{\prime}}^{3-i}\right) I\left(M_{\tau_{i}^{\prime}}^{3-i}>b_{3-i}\right) I\left(\tau_{i}^{\prime} \leq T^{\prime}\right)\right] \\
& =E_{x_{1}, m_{1}, x_{2}, m_{2}}\left[D_{1, i}\left(t+\tau_{i, 0}, b_{i}, X_{\tau_{i, 0}}^{3-i, 0}\right) I\left(M_{\tau_{i, 0}}^{3-i, 0}>b_{3-i}\right) I\left(\tau_{i, 0} \leq T^{\prime}\right)\right]
\end{aligned}
$$

for $x_{i} \geq m_{i}>b_{i}>0$, where $\tau_{i}^{\prime}=\tau_{i}\left(x_{1}, m_{1}, x_{2}, m_{2}\right)$ and the processes $\left(X^{3-i, 0}, M^{3-i, 0}\right)$ as well as the hitting times $\tau_{i, 0}, i=1,2$, are defined in (2.12)-(2.13) and (2.16) above. Therefore, taking into account the independence of $\tau_{i, 0}$ and $\left(X^{3-i, 0}, M^{3-i, 0}\right)$, from (3.4) and (3.5) we conclude that:

$$
\begin{aligned}
& E_{x_{1}, m_{1}, x_{2}, m_{2}}\left[D_{1, i}\left(\tau_{i}, X_{\tau_{i}}^{i}, X_{\tau_{i}}^{3-i}\right) I\left(t<\tau_{i} \leq T, \tau_{i}<\tau_{3-i}\right) \mid \mathcal{G}_{t}\right] \\
& =I\left(t<\tau_{1} \wedge \tau_{2}\right) \int_{0}^{T^{\prime}} \int_{b_{3-i}}^{\infty} \int_{b_{3-i}}^{\infty} D_{1, i}\left(t+u, b_{i}, x_{3-i}^{\prime}\right) h_{i, 0}\left(X_{t}^{i} ; u\right) \\
& \quad \times g_{3-i, 0}\left(X_{t}^{3-i}, M_{t}^{3-i} ; u, x_{3-i}^{\prime}, m_{3-i}^{\prime}\right) d u d x_{3-i}^{\prime} d m_{3-i}^{\prime}
\end{aligned}
$$

where the functions $g_{i, 0}$ and $h_{i, 0}, i=1,2$, are given in (2.15) and (2.18) above.

Summarizing the facts proved above let us now formulate the following assertion.

Theorem 3.1. The ex-dividend price of the first-to-default option (2.3) in the model with full information is given by the sum of the terms (3.3) and (3.6).

3.2. Let us continue by computing the terms for the second-to-default (2.4). For this, 
applying the Markov property of the process $\left(X^{1}, M^{1}, X^{2}, M^{2}\right)$, we get:

$$
\begin{aligned}
& E_{x_{1}, m_{1}, x_{2}, m_{2}}\left[\left(D_{2, i}\left(\tau_{i}, X_{\tau_{i}}^{i}, X_{\tau_{i}}^{3-i}\right)+C_{i}\left(X_{T}^{i}, X_{T}^{3-i}\right)\right) I\left(\tau_{3-i}<\tau_{i} \leq T\right) \mid \mathcal{G}_{t}\right] \\
& =E_{x_{1}, m_{1}, x_{2}, m_{2}}\left[\left(D_{2, i}\left(\tau_{i}, b_{i}, X_{\tau_{i}}^{3-i}\right)+C_{i}\left(X_{T}^{i}, X_{T}^{3-i}\right)\right) I\left(\tau_{3-i}<\tau_{i} \leq t\right) \mid \mathcal{G}_{t}\right] \\
& \quad+E_{x_{1}, m_{1}, x_{2}, m_{2}}\left[\left(D_{2, i}\left(\tau_{i}, b_{i}, X_{\tau_{i}}^{3-i}\right)+C_{i}\left(X_{T}^{i}, X_{T}^{3-i}\right)\right) I\left(\tau_{3-i} \leq t<\tau_{i} \leq T\right) \mid \mathcal{G}_{t}\right] \\
& \quad+E_{x_{1}, m_{1}, x_{2}, m_{2}}\left[\left(D_{2, i}\left(\tau_{i}, b_{i}, X_{\tau_{i}}^{3-i}\right)+C_{i}\left(X_{T}^{i}, X_{T}^{3-i}\right)\right) I\left(t<\tau_{3-i}<\tau_{i} \leq T\right) \mid \mathcal{G}_{t}\right] \\
& =I\left(\tau_{3-i}<\tau_{i} \leq t\right)\left(D_{2, i}\left(\tau_{i}, b_{i}, X_{\tau_{i}}^{3-i}\right)+E_{X_{t}^{1}, M_{t}^{1}, X_{t}^{2}, M_{t}^{2}}\left[C_{i}\left(X_{T^{\prime}}^{i}, X_{T^{\prime}}^{3-i}\right)\right]\right) \\
& \quad+I\left(\tau_{3-i} \leq t<\tau_{i}\right) E_{X_{t}^{1}, M_{t}^{1}, X_{t}^{2}, M_{t}^{2}}\left[\left(D_{2, i}\left(t+\tau_{i}^{\prime}, b_{i}, X_{\tau_{i}^{\prime}}^{3-i}\right)+C_{i}\left(X_{T^{\prime}}^{i}, X_{T^{\prime}}^{3-i}\right)\right) I\left(\tau_{i}^{\prime} \leq T^{\prime}\right)\right] \\
& \quad+I\left(t<\tau_{1} \wedge \tau_{2}\right) E_{X_{t}^{1}, M_{t}^{1}, X_{t}^{2}, M_{t}^{2}}\left[\left(D_{2, i}\left(t+\tau_{i}^{\prime}, b_{i}, X_{\tau_{i}}^{3-i}\right)+C_{i}\left(X_{T^{\prime}}^{i}, X_{T^{\prime}}^{3-i}\right)\right) I\left(\tau_{3-i}^{\prime}<\tau_{i}^{\prime} \leq T^{\prime}\right)\right]
\end{aligned}
$$

for all $0 \leq t \leq T$, and continue by computing each of the terms separately.

Firstly, we see that:

$$
E_{x_{1}, m_{1}, x_{2}, m_{2}}\left[C_{i}\left(X_{T^{\prime}}^{i}, X_{T^{\prime}}^{3-i}\right)\right]=E_{x_{1}, m_{1}, x_{2}, m_{2}}\left[C_{i}\left(X_{T^{\prime}}^{i, 3}, X_{T^{\prime}}^{3-i, 3}\right)\right]
$$

for $x_{i} \geq m_{i}$ with $b_{i} \geq m_{i}>0$, where the processes $\left(X^{i, 3}, M^{i, 3}\right), i=1,2$, are defined in (2.12)-(2.13) above. Then, using the independence of $\left(X^{i, 3}, M^{i, 3}\right)$ and $\left(X^{3-i, 3}, M^{3-i, 3}\right)$, we have:

$$
\begin{aligned}
& E_{x_{1}, m_{1}, x_{2}, m_{2}}\left[C_{i}\left(X_{T}^{i}, X_{T}^{3-i}\right) I\left(\tau_{3-i}<\tau_{i} \leq t\right) \mid \mathcal{G}_{t}\right] \\
& =I\left(\tau_{3-i}<\tau_{i} \leq t\right) \int_{0}^{\infty} \int_{0}^{b_{i}} \int_{0}^{\infty} \int_{0}^{b_{3-i}} C_{i}\left(x_{i}^{\prime}, x_{3-i}^{\prime}\right) \prod_{\ell=i}^{3-i} g_{\ell, 3}\left(X_{t}^{\ell}, M_{t}^{\ell} ; T-t, x_{\ell}^{\prime}, m_{\ell}^{\prime}\right) d x_{\ell}^{\prime} d m_{\ell}^{\prime}
\end{aligned}
$$

where the functions $g_{i, 3}, i=1,2$, are defined in (2.15) above.

Secondly, we observe that:

$$
\begin{aligned}
& E_{x_{1}, m_{1}, x_{2}, m_{2}}\left[D_{2, i}\left(t+\tau_{i}^{\prime}, b_{i}, X_{\tau_{i}^{\prime}}^{3-i}\right) I\left(\tau_{i}^{\prime} \leq T^{\prime}\right)\right] \\
& =E_{x_{1}, m_{1}, x_{2}, m_{2}}\left[D_{2, i}\left(t+\tau_{i, 3-i}, b_{i}, X_{\tau_{i, 3-i}}^{3-i, 3-i}\right) I\left(\tau_{i, 3-i} \leq T^{\prime}\right)\right]
\end{aligned}
$$

for $x_{i} \geq m_{i}>b_{i}>0$ and $x_{3-i} \geq m_{3-i}$ with $b_{3-i} \geq m_{3-i}>0$, where the processes $\left(X^{3-i, 3-i}, M^{3-i, 3-i}\right)$ as well as the hitting times $\tau_{i, 3-i}, i=1,2$, are defined in (2.12)-(2.13) and (2.16) above. Then, taking into account the independence of $\tau_{i, 3-i}$ and $\left(X^{3-i, 3-i}, M^{3-i, 3-i}\right)$, 
we get:

$$
\begin{aligned}
& E_{x_{1}, m_{1}, x_{2}, m_{2}}\left[D_{2, i}\left(t+\tau_{i}, b_{i}, X_{\tau_{i}}^{3-i}\right) I\left(\tau_{3-i} \leq t<\tau_{i} \leq T\right) \mid \mathcal{G}_{t}\right] \\
& =I\left(\tau_{3-i} \leq t<\tau_{i}\right) \int_{0}^{T-t} \int_{0}^{\infty} \int_{0}^{b_{3-i}} D_{2, i}\left(t+u, b_{i}, x_{3-i}^{\prime}\right) h_{i, 3-i}\left(X_{t}^{i} ; u\right) \\
& \quad \times g_{3-i, 3-i}\left(X_{t}^{3-i}, M_{t}^{3-i} ; u, x_{3-i}^{\prime}, m_{3-i}^{\prime}\right) d u d x_{3-i}^{\prime} d m_{3-i}^{\prime}
\end{aligned}
$$

where the functions $g_{3-i, 3-i}$ and $h_{i, 3-i}, i=1,2$, are defined in (2.15) and (2.18) above.

Thirdly, applying the strong Markov property of $\left(X^{1}, M^{1}, X^{2}, M^{2}\right)$, we get:

$$
\begin{aligned}
& E_{x_{1}, m_{1}, x_{2}, m_{2}}\left[C_{i}\left(X_{T^{\prime}}^{i}, X_{T^{\prime}}^{3-i}\right) I\left(\tau_{i}^{\prime} \leq T^{\prime}\right)\right] \\
& =E_{x_{1}, m_{1}, x_{2}, m_{2}}\left[\widehat{C}_{i}\left(X_{\tau_{i}^{\prime}}^{i}, M_{\tau_{i}^{\prime}}^{i}, X_{\tau_{i}^{\prime}}^{3-i}, M_{\tau_{i}^{\prime}}^{3-i} ; T^{\prime}-\tau_{i}^{\prime}\right) I\left(\tau_{i}^{\prime} \leq T^{\prime}\right)\right] \\
& =E_{x_{1}, m_{1}, x_{2}, m_{2}}\left[\widehat{C}_{i}\left(b_{i}, b_{i}, X_{\tau_{i, 3-i}}^{3-i, 3-i}, M_{\tau_{i, 3-i}}^{3-i, 3-i} ; T^{\prime}-\tau_{i, 3-i}\right) I\left(\tau_{i, 3-i} \leq T^{\prime}\right)\right]
\end{aligned}
$$

for $x_{i} \geq m_{i}>b_{i}>0$ and $x_{3-i} \geq m_{3-i}$ with $b_{3-i} \geq m_{3-i}>0$, where the functions $\widehat{C}_{i}, i=1,2$, are defined by:

$$
\begin{aligned}
\widehat{C}_{i}\left(x_{i}, m_{i}, x_{3-i}, m_{3-i} ; T^{\prime}-u\right) & =E_{x_{1}, m_{1}, x_{2}, m_{2}}\left[C_{i}\left(X_{T^{\prime}-u}^{i}, X_{T^{\prime}-u}^{3-i}\right)\right] \\
& =E_{x_{1}, m_{1}, x_{2}, m_{2}}\left[C_{i}\left(X_{T^{\prime}-u}^{i, 3}, X_{T^{\prime}-u}^{3-i, 3}\right)\right]
\end{aligned}
$$

for $x_{\ell} \geq m_{\ell}$ with $b_{\ell} \geq m_{\ell}>0, \ell=1,2$, and any $0 \leq u \leq T^{\prime}$ fixed. Thus, using the independence of $\tau_{i, 3-i}$ and $\left(X^{3-i, 3-i}, M^{3-i, 3-i}\right)$, from (3.12) we obtain:

$$
\begin{aligned}
& E_{x_{1}, m_{1}, x_{2}, m_{2}}\left[C_{i}\left(X_{T}^{i}, X_{T}^{3-i}\right) I\left(\tau_{3-i} \leq t<\tau_{i} \leq T\right)\right] \\
& \begin{aligned}
=I\left(\tau_{3-i} \leq t<\tau_{i}\right) \int_{0}^{T-t} \int_{0}^{\infty} \int_{0}^{b_{3-i}} \widehat{C}_{i}\left(b_{i}, b_{i}, x_{3-i}^{\prime}, m_{3-i}^{\prime} ; T-t-u\right) h_{i, 3-i}\left(X_{t}^{i} ; u\right) \\
\quad \times g_{3-i, 3-i}\left(X_{t}^{3-i}, M_{t}^{3-i} ; u, x_{3-i}^{\prime}, m_{3-i}^{\prime}\right) d u d x_{3-i}^{\prime} d m_{3-i}^{\prime}
\end{aligned}
\end{aligned}
$$

where, by virtue of independence of $\left(X^{i, 3}, M^{i, 3}\right)$ and $\left(X^{3-i, 3}, M^{3-i, 3}\right)$, from (3.13) it follows that:

$$
\begin{aligned}
& \widehat{C}_{i}\left(x_{i}^{\prime}, m_{i}^{\prime}, x_{3-i}^{\prime}, m_{3-i}^{\prime} ; T-t-u\right) \\
& =\int_{0}^{\infty} \int_{0}^{b_{i}} \int_{0}^{\infty} \int_{0}^{b_{3-i}} C_{i}\left(x_{i}^{\prime \prime}, x_{3-i}^{\prime \prime}\right) \prod_{\ell=i}^{3-i} g_{\ell, 3}\left(x_{\ell}^{\prime}, m_{\ell}^{\prime} ; T-t-u, x_{\ell}^{\prime \prime}, m_{\ell}^{\prime \prime}\right) d x_{\ell}^{\prime \prime} d m_{\ell}^{\prime \prime}
\end{aligned}
$$


and the functions $g_{i, \ell}$ and $h_{i, \ell}, i=1,2, \ell=1,2,3$, are defined in (2.15) and (2.18) above.

Now, applying the strong Markov property of $\left(X^{1}, M^{1}, X^{2}, M^{2}\right)$, we get:

$$
\begin{aligned}
& E_{x_{1}, m_{1}, x_{2}, m_{2}}\left[D_{2, i}\left(t+\tau_{i}^{\prime}, b_{i}, X_{\tau_{i}^{\prime}}^{3-i}\right) I\left(\tau_{3-i}^{\prime}<\tau_{i}^{\prime} \leq T^{\prime}\right)\right] \\
& =E_{x_{1}, m_{1}, x_{2}, m_{2}}\left[D_{2, i}\left(t+\tau_{i}^{\prime}, b_{i}, X_{\tau_{i}^{\prime}}^{3-i}\right) I\left(M_{\tau_{3-i}^{\prime}}^{i}>b_{i}\right) I\left(\tau_{i}^{\prime} \leq T^{\prime}\right)\right] \\
& =E_{x_{1}, m_{1}, x_{2}, m_{2}}\left[\widehat{D}_{2, i}\left(t+\tau_{3-i}^{\prime}, X_{\tau_{3-i}^{\prime}}^{i}, M_{\tau_{3-i}^{\prime}}^{i}, X_{\tau_{3-i}^{\prime}}^{3-i}, M_{\tau_{3-i}^{\prime}}^{3-i} ; T^{\prime}-\tau_{3-i}^{\prime}\right) I\left(M_{\tau_{3-i}^{\prime}}^{i}>b_{i}\right) I\left(\tau_{3-i}^{\prime} \leq T^{\prime}\right)\right] \\
& =E_{x_{1}, m_{1}, x_{2}, m_{2}}\left[\widehat{D}_{2, i}\left(t+\tau_{3-i, 0}, X_{\tau_{3-i, 0}}^{i, 0}, M_{\tau_{3-i, 0}}^{i, 0}, b_{3-i}, b_{3-i} ; T^{\prime}-\tau_{3-i, 0}\right) I\left(M_{\tau_{3-i, 0}}^{i, 0}>b_{i}\right) I\left(\tau_{3-i, 0} \leq T^{\prime}\right)\right]
\end{aligned}
$$

for $x_{i} \geq m_{i}>b_{i}>0$, where the functions $\widehat{D}_{2, i}, i=1,2$, are defined by:

$$
\begin{aligned}
& \widehat{D}_{2, i}\left(t+v, x_{i}, m_{i}, x_{3-i}, m_{3-i} ; T^{\prime}-v\right) \\
& =E_{x_{1}, m_{1}, x_{2}, m_{2}}\left[D_{2, i}\left(t+v+\tau_{i}^{\prime}, b_{i}, X_{\tau_{i}^{\prime}}^{3-i}\right) I\left(\tau_{i}^{\prime} \leq T^{\prime}-v\right)\right] \\
& =E_{x_{1}, m_{1}, x_{2}, m_{2}}\left[D_{2, i}\left(t+v+\tau_{i, 3-i}, b_{i}, X_{\tau_{i, 3-i}}^{3-i, 3-i}\right) I\left(\tau_{i, 3-i} \leq T^{\prime}-v\right)\right]
\end{aligned}
$$

for $x_{i} \geq m_{i}>b_{i}>0$ and $x_{3-i} \geq m_{3-i}$ with $b_{3-i} \geq m_{3-i}>0$, and any $0 \leq v \leq T^{\prime}$ fixed. Hence, using the independence of $\tau_{3-i, 0}$ and $\left(X^{i, 0}, M^{i, 0}\right)$, from (3.16) we obtain:

$$
\begin{gathered}
E_{x_{1}, m_{1}, x_{2}, m_{2}}\left[D_{2, i}\left(\tau_{i}, b_{i}, X_{\tau_{i}}^{3-i}\right) I\left(t<\tau_{3-i}<\tau_{i} \leq T\right) \mid \mathcal{G}_{t}\right] \\
=I\left(t<\tau_{1} \wedge \tau_{2}\right) \int_{0}^{T-t} \int_{0}^{\infty} \int_{b_{i}}^{\infty} \widehat{D}_{2, i}\left(t+v, x_{i}^{\prime}, m_{i}^{\prime}, b_{3-i}, b_{3-i} ; T-t-v\right) h_{3-i, 0}\left(X_{t}^{3-i} ; v\right) \\
\times g_{i, 0}\left(X_{t}^{i}, M_{t}^{i} ; v, x_{i}^{\prime}, m_{i}^{\prime}\right) d v d x_{i}^{\prime} d m_{i}^{\prime}
\end{gathered}
$$

where, by virtue of the independence of $\tau_{i, 3-i}$ and $\left(X^{3-i, 3-i}, M^{3-i, 3-i}\right)$, from (3.17) it follows that:

$$
\begin{aligned}
& \widehat{D}_{2, i}\left(t+v, x_{i}^{\prime}, m_{i}^{\prime}, x_{3-i}^{\prime}, m_{3-i}^{\prime} ; T-t-v\right) \\
& =\int_{0}^{T-t-v} \int_{0}^{\infty} \int_{0, i}^{b_{3-i}} D_{2, i}\left(t+v+u, b_{i}, x_{3-i}^{\prime \prime}\right) h_{i, 3-i}\left(x_{i}^{\prime} ; u\right) \\
& \quad \times g_{3-i, 3-i}\left(x_{3-i}^{\prime}, m_{3-i}^{\prime} ; u, x_{3-i}^{\prime \prime}, m_{3-i}^{\prime \prime}\right) d u d x_{3-i}^{\prime \prime} d m_{3-i}^{\prime \prime}
\end{aligned}
$$

and the functions $g_{i, \ell}$ and $h_{i, \ell}, i=1,2, \ell=0,1,2$, are defined in (2.15) and (2.18) above. 
Finally, we see that:

$$
\begin{aligned}
& E_{x_{1}, m_{1}, x_{2}, m_{2}}\left[C_{i}\left(X_{T^{\prime}}^{i}, X_{T^{\prime}}^{3-i}\right) I\left(\tau_{3-i}^{\prime}<\tau_{i}^{\prime} \leq T^{\prime}\right)\right] \\
& =E_{x_{1}, m_{1}, x_{2}, m_{2}}\left[C_{i}\left(X_{T^{\prime}}^{i}, X_{T^{\prime}}^{3-i}\right) I\left(M_{\tau_{3-i}^{\prime}}^{i}>b_{i}\right) I\left(\tau_{i}^{\prime} \leq T^{\prime}\right)\right] \\
& =E_{x_{1}, m_{1}, x_{2}, m_{2}}\left[\widetilde{C}_{i}\left(X_{\tau_{3-i}^{\prime}}^{i}, M_{\tau_{3-i}^{\prime}}^{i}, X_{\tau_{3-i}^{\prime}}^{3-i}, M_{\tau_{3-i}^{\prime}}^{3-i} ; T^{\prime}-\tau_{3-i}^{\prime}\right) I\left(M_{\tau_{3-i}^{\prime}}^{i}>b_{i}\right) I\left(\tau_{3-i}^{\prime} \leq T^{\prime}\right)\right] \\
& =E_{x_{1}, m_{1}, x_{2}, m_{2}}\left[\widetilde{C}_{i}\left(X_{\tau_{3-i, 0}}^{i}, M_{\tau_{3-i, 0}}^{i}, b_{3-i}, b_{3-i} ; T^{\prime}-\tau_{3-i, 0}\right) I\left(M_{\tau_{3-i, 0}}^{i}>b_{i}\right) I\left(\tau_{3-i, 0} \leq T^{\prime}\right)\right]
\end{aligned}
$$

for $x_{i} \geq m_{i}>b_{i}>0$, where the functions $\widetilde{C}_{i}, i=1,2$, are defined by:

$$
\begin{aligned}
& \widetilde{C}_{i}\left(x_{i}, m_{i}, x_{3-i}, m_{3-i} ; T^{\prime}-v\right) \\
& =E_{x_{1}, m_{1}, x_{2}, m_{2}}\left[\widehat{C}_{i}\left(X_{\tau_{i}^{\prime}}^{i}, M_{\tau_{i}^{\prime}}^{i}, X_{\tau_{i}^{\prime}}^{3-i}, M_{\tau_{i}^{\prime}}^{3-i} ; T^{\prime}-v-\tau_{i}^{\prime}\right) I\left(\tau_{i}^{\prime} \leq T^{\prime}-v\right)\right] \\
& =E_{x_{1}, m_{1}, x_{2}, m_{2}}\left[\widehat{C}_{i}\left(b_{i}, b_{i}, X_{\tau_{i, 3}-i}^{3-i, 3-i}, M_{\tau_{i, 3-i}}^{3-i, 3-i} ; T^{\prime}-v-\tau_{i, 3-i}\right) I\left(\tau_{i, 3-i} \leq T^{\prime}-v\right)\right]
\end{aligned}
$$

for $x_{i} \geq m_{i}>b_{i}>0$ and $x_{3-i} \geq m_{3-i}$ with $b_{3-i} \geq m_{3-i}>0$, and any $0 \leq v \leq T^{\prime}$ fixed, where the functions $\widehat{C}_{i}, i=1,2$, are defined in (3.13) above. Hence, using the independence of $\tau_{3-i, 0}$ and $\left(X^{i, 0}, M^{i, 0}\right)$, from (3.20) we obtain:

$$
\begin{gathered}
E_{x_{1}, m_{1}, x_{2}, m_{2}}\left[C_{i}\left(X_{T}^{i}, X_{T}^{3-i}\right) I\left(t<\tau_{3-i}<\tau_{i} \leq T\right) \mid \mathcal{G}_{t}\right] \\
\begin{aligned}
=I\left(t<\tau_{1} \wedge \tau_{2}\right) \int_{0}^{T-t} \int_{b_{i}}^{\infty} \int_{b_{i}}^{\infty} \widetilde{C}_{i}\left(x_{i}^{\prime}, m_{i}^{\prime}, b_{3-i}, b_{3-i} ; T-t-v\right) h_{3-i, 0}\left(X_{t}^{3-i} ; v\right) \\
\quad \times g_{i, 0}\left(X_{t}^{i}, M_{t}^{i} ; v, x_{i}^{\prime}, m_{i}^{\prime}\right) d v d x_{i}^{\prime} d m_{i}^{\prime}
\end{aligned}
\end{gathered}
$$

where, by virtue of the independence of $\tau_{i, 3-i}$ and $\left(X^{3-i, 3-i}, M^{3-i, 3-i}\right)$, from (3.21) it follows that:

$$
\begin{aligned}
& \widetilde{C}_{i}\left(x_{i}^{\prime}, m_{i}^{\prime}, x_{3-i}^{\prime}, m_{3-i}^{\prime} ; T-t-v\right) \\
& =\int_{0}^{T-t-v} \int_{0}^{\infty} \int_{0}^{b_{3-i}} \widehat{C}_{i}\left(b_{i}, b_{i}, x_{3-i}^{\prime \prime}, m_{3-i}^{\prime \prime} ; T-t-v-u\right) h_{i, 3-i}\left(x_{i}^{\prime} ; u\right) \\
& \quad \times g_{3-i, 3-i}\left(x_{3-i}^{\prime}, m_{3-i}^{\prime} ; u, x_{3-i}^{\prime \prime}, m_{3-i}^{\prime \prime}\right) d u d x_{3-i}^{\prime \prime} d m_{3-i}^{\prime \prime}
\end{aligned}
$$

the functions $\widehat{C}_{i}$ admit the representation (3.15) and the functions $g_{i, \ell}$ and $h_{i, \ell}, i=1,2$, $\ell=0,1,2$, are defined in (2.15) and (2.18) above.

Therefore, summarizing the facts proved above we are now ready to formulate the following assertion. 
Theorem 3.2. The ex-dividend price of the second-to-default option (2.4) in the model with full information is given by the sum of (3.9), (3.11), (3.14), (3.18) and (3.22).

\section{Case of partial information}

In this section we compute the conditional expectations (2.5) and (2.6).

4.1. Let us proceed by computing the terms for the first-to-default (2.5). For this, let $H\left(x_{j}, m_{j}, x_{3-j}, m_{3-j}\right)$ be a nonnegative continuous function for any $j=1,2$ fixed. By virtue of independence of the processes $\left(X^{j, 0}, M^{j, 0}\right)$ and $\left(X^{3-j, 0}, M^{3-j, 0}\right)$ defined in (2.12)-(2.13), we get:

$$
\begin{aligned}
& E_{x_{1}, m_{1}, x_{2}, m_{2}}\left[H\left(X_{t}^{j}, M_{t}^{j}, X_{t}^{3-j}, M_{t}^{3-j}\right) I\left(t<\tau_{j} \wedge \tau_{3-j}\right) \mid \mathcal{G}_{t}^{j}\right] \\
& =I\left(t<\tau_{j}\right) E_{x_{1}, m_{1}, x_{2}, m_{2}}\left[H\left(X_{t}^{j, 0}, M_{t}^{j, 0}, X_{t}^{3-j, 0}, M_{t}^{3-j, 0}\right) I\left(M_{t}^{3-j, 0}>b_{3-j}\right) \mid \mathcal{G}_{t}^{j}\right] \\
& =I\left(t<\tau_{j}\right) \int_{b_{3-j}}^{\infty} \int_{b_{3-j}}^{\infty} H\left(X_{t}^{j, 0}, M_{t}^{j, 0}, x_{3-j}^{\prime}, m_{3-j}^{\prime}\right) g_{3-j, 0}\left(x_{3-j}, m_{3-j} ; t, x_{3-j}^{\prime}, m_{3-j}^{\prime}\right) d x_{3-j}^{\prime} d m_{3-j}^{\prime}
\end{aligned}
$$

for all $0 \leq t \leq T$, where the functions $g_{3-j, 0}, j=1,2$, are defined in (2.15) above.

Now, we see that:

$$
\begin{aligned}
& E_{x_{1}, m_{1}, x_{2}, m_{2}}\left[D_{1, j}\left(\tau_{j}, X_{\tau_{j}}^{j}, X_{\tau_{j}}^{3-j}\right) I\left(\tau_{j} \leq t, \tau_{j}<\tau_{3-j}\right) \mid \mathcal{G}_{t}^{j}\right] \\
& =I\left(\tau_{j} \leq t\right) E_{x_{1}, m_{1}, x_{2}, m_{2}}\left[D_{1, j}\left(\tau_{j}, b_{j}, X_{\tau_{j}}^{3-j}\right) I\left(M_{\tau_{j}}^{3-j}>b_{3-j}\right) \mid \mathcal{G}_{t}^{j}\right] \\
& =I\left(\tau_{j} \leq t\right) E_{x_{1}, m_{1}, x_{2}, m_{2}}\left[D_{1, j}\left(\tau_{j, 0}, b_{j}, X_{\tau_{j, 0}}^{3-j, 0}\right) I\left(M_{\tau_{j, 0}}^{3-j, 0}>b_{3-j}\right) \mid \mathcal{G}_{t}^{j}\right]
\end{aligned}
$$

for $0 \leq t \leq T$, where the hitting times $\tau_{j, 0}, j=1,2$, are defined in (2.16). Thus, using the independence of $\tau_{j, 0}$ and $\left(X^{3-j, 0}, M^{3-j, 0}\right)$, we have:

$$
\begin{aligned}
& E_{x_{1}, m_{1}, x_{2}, m_{2}}\left[D_{1, j}\left(\tau_{j}, X_{\tau_{j}}^{j}, X_{\tau_{j}}^{3-j}\right) I\left(\tau_{j} \leq t, \tau_{j}<\tau_{3-j}\right) \mid \mathcal{G}_{t}^{j}\right] \\
& =I\left(\tau_{j} \leq t\right) \int_{b_{3-j}}^{\infty} \int_{b_{3-j}}^{\infty} D_{1, j}\left(\tau_{j}, b_{j}, x_{3-j}^{\prime}\right) g_{3-j, 0}\left(x_{3-j}, m_{3-j} ; \tau_{j}, x_{3-j}^{\prime}, m_{3-j}^{\prime}\right) d x_{3-j}^{\prime} d m_{3-j}^{\prime}
\end{aligned}
$$

where the functions $g_{3-j, 0}, j=1,2$, are defined in (2.15) above.

Then, we observe that:

$$
\begin{aligned}
& E_{x_{1}, m_{1}, x_{2}, m_{2}}\left[D_{1, j}\left(\tau_{j}, X_{\tau_{j}}^{j}, X_{\tau_{j}}^{3-j}\right) I\left(\tau_{j} \leq t, \tau_{j}<\tau_{3-j}\right) \mid \mathcal{G}_{t}^{3-j}\right] \\
& =E_{x_{1}, m_{1}, x_{2}, m_{2}}\left[D_{1, j}\left(\tau_{j}, b_{j}, X_{\tau_{j}}^{3-j}\right) I\left(\tau_{j}<\tau_{3-j} \leq t\right) \mid \mathcal{G}_{t}^{3-j}\right] \\
& \quad+E_{x_{1}, m_{1}, x_{2}, m_{2}}\left[D_{1, j}\left(\tau_{j}, b_{j}, X_{\tau_{j}}^{3-j}\right) I\left(\tau_{j} \leq t<\tau_{3-j}\right) \mid \mathcal{G}_{t}^{3-j}\right]
\end{aligned}
$$


for $0 \leq t \leq T$ and $j=1,2$. Hence, by virtue of the independence of $\tau_{j, 0}$ and $\left(X^{3-j, 0}, M^{3-j, 0}\right)$ we obtain:

$$
\begin{aligned}
& E_{x_{1}, m_{1}, x_{2}, m_{2}}\left[D_{1, j}\left(\tau_{j}, b_{j}, X_{\tau_{j}}^{3-j}\right) I\left(\tau_{j}<\tau_{3-j} \leq t\right) \mid \mathcal{G}_{t}^{3-j}\right] \\
& =I\left(\tau_{3-j} \leq t\right) E_{x_{1}, m_{1}, x_{2}, m_{2}}\left[D_{1, j}\left(\tau_{j}, b_{j}, X_{\tau_{j}}^{3-j}\right) I\left(M_{\tau_{j}}^{3-j}>b_{3-j}\right) I\left(\tau_{j}<\tau_{3-j}\right) \mid \mathcal{G}_{t}^{3-j}\right] \\
& =I\left(\tau_{3-j} \leq t\right) E_{x_{1}, m_{1}, x_{2}, m_{2}}\left[D_{1, j}\left(\tau_{j, 0}, b_{j}, X_{\tau_{j, 0}}^{3-j, 0}\right) I\left(M_{\tau_{j, 0}}^{3-j, 0}>b_{3-j}\right) I\left(\tau_{j, 0}<\tau_{3-j}\right) \mid \mathcal{G}_{t}^{3-j}\right]
\end{aligned}
$$

and

$$
\begin{aligned}
& E_{x_{1}, m_{1}, x_{2}, m_{2}}\left[D_{1, j}\left(\tau_{j}, b_{j}, X_{\tau_{j}}^{3-j}\right) I\left(\tau_{j} \leq t<\tau_{3-j}\right) \mid \mathcal{G}_{t}^{3-j}\right] \\
& =I\left(t<\tau_{3-j}\right) E_{x_{1}, m_{1}, x_{2}, m_{2}}\left[D_{1, j}\left(\tau_{j}, b_{j}, X_{\tau_{j}}^{3-j}\right) I\left(M_{\tau_{j}}^{3-j}>b_{3-j}\right) I\left(\tau_{j} \leq t\right) \mid \mathcal{G}_{t}^{3-j}\right] \\
& =I\left(t<\tau_{3-j}\right) E_{x_{1}, m_{1}, x_{2}, m_{2}}\left[D_{j, 1}\left(\tau_{j, 0}, b_{j}, X_{\tau_{j, 0}}^{3-j, 0}\right) I\left(M_{\tau_{j, 0}}^{3-j, 0}>b_{3-j}\right) I\left(\tau_{j, 0} \leq t\right) \mid \mathcal{G}_{t}^{3-j}\right]
\end{aligned}
$$

and thus, from (4.4) we conclude that:

$$
\begin{aligned}
& E_{x_{1}, m_{1}, x_{2}, m_{2}}\left[D_{1, j}\left(\tau_{j}, b_{j}, X_{\tau_{j}}^{3-j}\right) I\left(\tau_{j} \leq t, \tau_{j}<\tau_{3-j}\right) \mid \mathcal{G}_{t}^{3-j}\right] \\
& =\int_{0}^{\tau_{3-j} \wedge t} D_{1, j}\left(u, b_{j}, X_{u}^{3-j}\right) I\left(M_{u}^{3-j}>b_{3-j}\right) h_{j, 0}\left(x_{j} ; u\right) d u
\end{aligned}
$$

where the functions $h_{j, 0}, j=1,2$, are defined in (2.18) above.

Summarizing the facts proved above let us formulate the following assertion.

Theorem 4.1. The ex-dividend price of first-to-default option (2.5) in the model with partial information is given by the sum of (4.1) and (4.3) or (4.7), where the function $H$ is given appropriately by the corresponding value in (3.3) or (3.6), respectively.

4.2. Let us conclude by computing the terms for the second-to-default (2.6). Firstly, taking into account the Markovian structure of the process $\left(X^{1}, M^{1}, X^{2}, M^{2}\right)$, we get:

$$
\begin{aligned}
& E_{x_{1}, m_{1}, x_{2}, m_{2}}\left[H\left(X_{t}^{j}, M_{t}^{j}, X_{t}^{3-j}, M_{t}^{3-j}\right) I\left(\tau_{3-j} \leq t<\tau_{j}\right) \mid \mathcal{G}_{t}^{j}\right] \\
& =I\left(t<\tau_{j}\right) \int_{0}^{t} \int_{0}^{\infty} \int_{0}^{b_{3-j}} H\left(X_{t}^{j}, M_{t}^{j}, x_{3-j}^{\prime}, m_{3-j}^{\prime}\right) h_{3-j, 0}\left(x_{3-j} ; u\right) \\
& \quad \times g_{3-j, 3-j}\left(b_{3-j}, b_{3-j} ; t-u, x_{3-j}^{\prime}, m_{3-j}^{\prime}\right) d u d x_{3-j}^{\prime} d m_{3-j}^{\prime}
\end{aligned}
$$

and

$$
\begin{aligned}
& E_{x_{1}, m_{1}, x_{2}, m_{2}}\left[H\left(X_{t}^{j}, M_{t}^{j}, X_{t}^{3-j}, M_{t}^{3-j}\right) I\left(\tau_{3-j} \leq t<\tau_{j}\right) \mid \mathcal{G}_{t}^{3-j}\right] \\
& =I\left(\tau_{3-j} \leq t\right) \int_{b_{j}}^{\infty} \int_{b_{j}}^{\infty} \widehat{H}\left(x_{j}^{\prime}, m_{j}^{\prime}, X_{t}^{3-j}, M_{t}^{3-j} ; t-\tau_{3-j}\right) g_{j, 0}\left(x_{j}, m_{j} ; \tau_{3-j}, x_{j}^{\prime}, m_{j}^{\prime}\right) d x_{j}^{\prime} d m_{j}^{\prime}
\end{aligned}
$$


where the function $\widehat{H}$ is defined by:

$$
\begin{aligned}
& \widehat{H}\left(x_{j}^{\prime}, m_{j}^{\prime}, x_{3-j}^{\prime}, m_{3-j}^{\prime} ; t-v\right) \\
& =\int_{b_{j}}^{\infty} \int_{b_{j}}^{\infty} H\left(x_{j}^{\prime \prime}, m_{j}^{\prime \prime}, x_{3-j}^{\prime}, m_{3-j}^{\prime}\right) g_{j, 3-j}\left(x_{j}^{\prime}, m_{j}^{\prime} ; t-v, x_{j}^{\prime \prime}, m_{j}^{\prime \prime}\right) d x_{j}^{\prime \prime} d m_{j}^{\prime \prime}
\end{aligned}
$$

for $0 \leq t \leq T$ and the functions $g_{j, \ell}, j=1,2, \ell=0,1,2$, are defined in (2.15) above.

Now, by virtue of Markovian structure of the process $\left(X^{1}, M^{1}, X^{2}, M^{2}\right)$, we see that:

$$
\begin{aligned}
& E_{x_{1}, m_{1}, x_{2}, m_{2}}\left[D_{2, j}\left(\tau_{j}, b_{j}, X_{\tau_{j}}^{3-j}\right) I\left(\tau_{3-j}<\tau_{j} \leq t\right) \mid \mathcal{G}_{t}^{j}\right] \\
& =I\left(\tau_{j} \leq t\right) \int_{0}^{\tau_{j}} \int_{0}^{\infty} \int_{0}^{b_{3-j}} D_{2, j}\left(\tau_{j}, b_{j}, x_{3-j}^{\prime}\right) h_{3-j, 0}\left(x_{3-j} ; u\right) \\
& \quad \times g_{3-j, 3-j}\left(b_{3-j}, b_{3-j} ; \tau_{j}-u, x_{3-j}^{\prime}, m_{3-j}^{\prime}\right) d u d x_{3-j}^{\prime} d m_{3-j}^{\prime}
\end{aligned}
$$

and

$$
\begin{aligned}
& E_{x_{1}, m_{1}, x_{2}, m_{2}}\left[D_{2, j}\left(\tau_{j}, b_{j}, X_{\tau_{j}}^{3-j}\right) I\left(\tau_{3-j}<\tau_{j} \leq t\right) \mid \mathcal{G}_{t}^{3-j}\right] \\
& =I\left(\tau_{3-j} \leq t\right) \int_{\tau_{3-j}}^{t} \int_{b_{j}}^{\infty} \int_{b_{j}}^{\infty} D_{2, j}\left(u, b_{j}, X_{u}^{3-j}\right) h_{j, 3-j}\left(x_{j}^{\prime} ; u-\tau_{3-j}\right) \\
& \quad \times g_{j, 0}\left(x_{j}, m_{j} ; \tau_{3-j}, x_{j}^{\prime}, m_{j}^{\prime}\right) d u d x_{j}^{\prime} d m_{j}^{\prime}
\end{aligned}
$$

for $0 \leq t \leq T$, where the functions $g_{j, \ell}$ and $h_{j, \ell}, j=1,2, \ell=0,1,2$, are defined in (2.15) and (2.18) above.

Finally, using again the Markovian structure of the process $\left(X^{1}, M^{1}, X^{2}, M^{2}\right)$, we obtain:

$$
\begin{aligned}
& E_{x_{1}, m_{1}, x_{2}, m_{2}}\left[H\left(X_{t}^{j}, M_{t}^{j}, X_{t}^{3-j}, M_{t}^{3-j}\right) I\left(\tau_{3-j}<\tau_{j} \leq t\right) \mid \mathcal{G}_{t}^{j}\right] \\
& =I\left(\tau_{j} \leq t\right) \int_{0}^{\tau_{j}} \int_{0}^{\infty} \int_{0}^{b_{3-j}} \widetilde{H}\left(X_{t}^{j}, M_{t}^{j}, x_{3-j}^{\prime}, m_{3-j}^{\prime} ; t-\tau_{j}\right) h_{3-j, 0}\left(x_{3-j} ; v\right) \\
& \quad \times g_{3-j, 3-j}\left(b_{3-j}, b_{3-j} ; \tau_{j}-v, x_{3-j}^{\prime}, m_{3-j}^{\prime}\right) d v d x_{3-j}^{\prime} d m_{3-j}^{\prime}
\end{aligned}
$$

where the function $\widetilde{H}$ is defined by:

$$
\begin{aligned}
& \widetilde{H}\left(x_{j}^{\prime}, m_{j}^{\prime}, x_{3-j}^{\prime}, m_{3-j}^{\prime} ; t-v\right) \\
& =\int_{0}^{\infty} \int_{0}^{b_{3-j}} H\left(x_{j}^{\prime}, m_{j}^{\prime}, x_{3-j}^{\prime \prime}, m_{3-j}^{\prime \prime}\right) g_{3-j, 3}\left(x_{j}^{\prime}, m_{j}^{\prime} ; t-v, x_{3-j}^{\prime \prime}, m_{3-j}^{\prime \prime}\right) d x_{3-j}^{\prime \prime} d m_{3-j}^{\prime \prime}
\end{aligned}
$$


and

$$
\begin{gathered}
E_{x_{1}, m_{1}, x_{2}, m_{2}}\left[H\left(X_{t}^{j}, M_{t}^{j}, X_{t}^{3-j}, M_{t}^{3-j}\right) I\left(\tau_{3-j}<\tau_{j} \leq t\right) \mid \mathcal{G}_{t}^{3-j}\right] \\
=I\left(\tau_{3-j} \leq t\right) \int_{\tau_{3-j}}^{t} \int_{b_{j}}^{\infty} \int_{b_{j}}^{\infty} \bar{H}\left(b_{j}, b_{j}, X_{t}^{3-j}, M_{t}^{3-j} ; t-u\right) h_{j, 3-j}\left(x_{j}^{\prime} ; u-\tau_{3-j}\right) \\
\quad \times g_{j, 0}\left(x_{j}, m_{j} ; \tau_{3-j}, x_{j}^{\prime}, m_{j}^{\prime}\right) d u d x_{j}^{\prime} d m_{j}^{\prime}
\end{gathered}
$$

where the function $\bar{H}$ is defined by:

$$
\begin{aligned}
& \bar{H}\left(x_{j}^{\prime}, m_{j}^{\prime}, x_{3-j}^{\prime}, m_{3-j}^{\prime} ; t-u\right) \\
& =\int_{0}^{\infty} \int_{0}^{b_{j}} H\left(x_{j}^{\prime \prime}, m_{j}^{\prime \prime}, x_{3-j}^{\prime}, m_{3-j}^{\prime}\right) g_{j, 3}\left(x_{j}^{\prime}, m_{j}^{\prime} ; t-u, x_{j}^{\prime \prime}, m_{j}^{\prime \prime}\right) d x_{j}^{\prime \prime} d m_{j}^{\prime \prime}
\end{aligned}
$$

for $0 \leq t \leq T$ and the functions $g_{j, \ell}$ and $h_{j, \ell}, j=1,2, \ell=0,1,2,3$, are defined in (2.15) and (2.18) above.

Therefore, summarizing the facts proved above we are now ready to formulate the following assertion.

Theorem 4.2. The ex-dividend price of first-to-default option (2.6) in the model with partial information is given by the sum of (4.1), (4.8), (4.9), (4.11), (4.12), (4.13) and (4.15), where the function $H$ is given appropriately by the corresponding value in (3.9), (3.14) or (3.22), respectively.

Acknowledgments. The paper was partially written when the first author was visiting Université d'Evry Val d'Essonne in May and November 2006, and in April 2008. The warm hospitality and financial support from Europlace Instutute of Finance and European Science Foundation (ESF) through the grant number 1356 of the programme Advanced Mathematical Methods for Finance (AMaMeF) are gratefully acknowledged.

\section{References}

[1] Bielecki, T. R. and Rutkowski, M. (2004). Credit Risk: Modeling, Valuation and Hedging. Springer, Berlin.

[2] Black, F. and Cox, J. C. (1976). Valuing corporate securities: Some effects of bond interdenture provisions. Journal of Finance 31 (351-367). 
[3] Borodin, A. N. and Salminen, P. (1996). Handbook of Brownian Motion. Birkhäuser, Basel.

[4] Geske, R. (1978). The pricing of options with stochastic dividend yield. Journal of Finance 33(2) (617-625).

[5] Giesecke, K. (2004). Correlated defaults with incomplete information. Journal of Banking and Finance 28 (1521-1545).

[6] He, H., Keirstead, W. P. and Rebholz, J. (1998). Double lookbacks. Mathematical Finance 8 (201-228).

[7] Iyengar, S. (2002). Hitting lines with two dimensional Brownian motion. SIAM Journal of Applied Mathematics 45 (983-989).

[8] Karatzas, I. and Shreve, S. E. (1998). Methods of Mathematical Finance. Springer, New York.

[9] Liptser, R. S. and Shiryaev, A. N. (1977). Statistics of Random Processes I. Springer, Berlin.

[10] Merton, R. (1974). On the pricing of corporate debt: the risk structure of interest rates. Journal of Finance 3 (449-470).

[11] Overbeck, L. and Schmidt, W. (2005). Modeling default dependence with threshold models. Journal of Derivatives 12 (10-19).

[12] Patras, F. (2006). A reflection principle for correlated defaults. Stochastic Processes and their Applications 116 (690-698).

[13] Revuz, D. and Yor, M. (1999). Continuous Martingales and Brownian Motion. Springer, Berlin.

[14] Schönbucher, P. J. (2003). Credit Derivatives Pricing Models: Models, Pricing and Implementation. Wiley, Chichester.

[15] Shiryaev, A. N. (1999). Essentials of Stochastic Finance. World Scientific, Singapore. 
[16] ValuŽIs, M. (2008). On the probabilities of correlated defaults: a first passage time approach. Nonlinear Analysis: Modeling and Control 13 (117-133).

[17] Zhou, C. (2001). An analysis of default correlations and multiple defaults. Review of Financial Studies 14 (555-576). 